\title{
Boehmians of type $S$ and their Fourier transforms
}

\begin{abstract}
Function spaces of type $S$ are introduced and investigated in the literature. They are also applied to study the Cauchy problem. In this paper we shall extend the concept of these spaces to the context of Boehmian spaces and study the Fourier transform theory on these spaces. These spaces enable us to combine the theory of Fourier transform on these function spaces as well as their dual spaces.
\end{abstract}

1. Introduction. In [3], function spaces of type $S$ are introduced and the properties of their Fourier transforms are investigated (see also [4], [5]). They are also applied to study the Cauchy problem. These spaces are constructed imposing conditions on the behavior of functions and on the growth of their derivatives at infinity as the order of the derivative increases (as is done in the case of generalized functions (see for example [9])). We shall first recall that the space $S_{\alpha}^{\beta}(\alpha \geq 0, \beta \geq 0)$ consists of all infinitely differentiable functions $\varphi(x)(-\infty<x<\infty)$ satisfying the inequalities

$$
\left|x^{k} \varphi^{(q)}(x)\right| \leq C A^{k} B^{q} k^{k \alpha} q^{q \beta},
$$

where the constants $A, B, C$ depend on the function $\varphi$. It is also known that for sufficiently small values of $\alpha$ and $\beta$ this space degenerates into the single function $\varphi(x) \equiv 0$. Hence in order to consider nontrivial spaces we shall be interested in choosing $\alpha$ and $\beta$ sufficiently large. Further spaces

2000 Mathematics Subject Classification. 44A40, 46A19, 46F10.

Key words and phrases. Boehmians, spaces of type $S$, Fourier transform. 
$S_{\alpha}, S^{\beta}, \mathscr{S}$ (here $\mathscr{S}$ denotes the usual space of rapidly decreasing functions) which can be described independently can be related to the space $S_{\alpha}^{\beta}$. For an interesting relationship between these spaces and examples we refer the reader to [3], [4] and [5]. It should be noted that for $\beta \leq 1$, functions in $S^{\beta}$ are already analytic in unbounded regions of the complex plane and hence cannot contain functions of bounded support. However for $\beta>1$ it is known (see [3]) that the space $S_{0}^{\beta}$ contains infinitely differentiable functions with compact support. Thus by virtue of the inequality $S_{0}^{\beta} \subset S_{\alpha}^{\beta}$ for $\alpha \geq 0$, we can safely assume that $S_{\alpha}^{\beta}(\beta>1)$ certainly contains infinitely differentiable functions with compact support. This fact will be crucial for our construction of the Boehmian space. Incidentally the Boehmian space we construct contains also a copy of the dual of $S_{\alpha}^{\beta}(\beta>1)$ (which is denoted as $\left(S_{\alpha}^{\beta}\right)^{\prime}$ ) and hence a study of Fourier transforms of elements of our Boehmian space generalizes the theory of Fourier transform on $\left(S_{\alpha}^{\beta}\right)^{\prime}$ which is undertaken in [3].

We shall assume the construction of Boehmian spaces and their properties (see [6], [7], [8]). In Section 2, we shall develop the preliminaries. In Section 2, we shall construct the Boehmian spaces. In Section 4, we shall define the Fourier transform and investigate its properties.

2. Preliminaries. In the following we shall define the required function spaces and obtain some properties. Fix $\alpha, \beta \geq 0$.

Definition 2.1. A function $f: \mathbb{R} \rightarrow \mathbb{C}$ is said to be in the family $G$ if it satisfies the following conditions:

(i) $f$ is an infinitely differentiable function.

(ii) $\left|f^{(k)}(x)\right| \leq c_{n, k} e^{\frac{1}{n}|x|^{1 / \alpha}}$ for all $n \in \mathbb{N}, x \in \mathbb{R}, k=0,1,2, \ldots$.

The topology on $G$ is given by the following countable separating family of semi-norms

$$
P_{n, k}(f)=\sup _{x \in \mathbb{R}, q \leq k}\left|f^{(q)}(x)\right| e^{\left(-\frac{1}{n}|x|^{1 / \alpha}\right)} \quad n=1,2,3, \ldots, k=0,1,2, \ldots
$$

It is easy to show that $G$ is a Fréchet space.

Definition 2.2. A function $\varphi: \mathbb{R} \rightarrow \mathbb{C}$ is said to be in $S_{\alpha}^{\beta}$ if

(i) $\varphi$ is an infinitely differentiable function.

(ii) $\left|x^{k} \varphi^{(q)}(x)\right| \leq C A^{k} B^{q} k^{k \alpha} q^{q \beta}(k=0,1,2, \ldots, q=0,1,2, \ldots)$ where $A, B$ and $C$ are constants depending on $\varphi$.

Here we assume that $k^{k \alpha}=1$ for $k=0$ and $q^{q \beta}=1$ for $q=0$.

From [3] it follows that the space $S_{\alpha}^{\beta}$ defined above is a countable union of Fréchet spaces which are denoted by $S_{\alpha, A}^{\beta, B}$ where $A, B=1,2, \ldots$ We also recall the following theorem from [1] and [2].

Theorem 2.3. If $\alpha+\beta \geq 1$, then the following are equivalent: 
(1) $\varphi \in S_{\alpha}^{\beta}$.

(2) $\sup _{x \in \mathbb{R}}|\varphi(x)| e^{\left(h|x|^{1 / \alpha}\right)}<\infty$ and $\sup _{t \in \mathbb{R}}|\hat{\varphi}(t)| e^{\left(k|t|^{1 / \beta}\right)}<\infty$ for some $h, k>0$.

Definition 2.4. A function $\varphi: \mathbb{R} \rightarrow \mathbb{C}$ is said to be in the space $T_{n, m}$ $(n, m \in \mathbb{N})$ if it satisfies the following conditions:

(i) $\varphi$ is infinitely differentiable.

(ii) $\|\varphi\|_{n, m}=\sup _{x \in \mathbb{R}}|\varphi(x)| e^{\left(\frac{1}{n}|x|^{1 / \alpha}\right)}+\sup _{t \in \mathbb{R}}|\hat{\varphi}(t)| e^{\left(\frac{1}{m}|t|^{1 / \beta}\right)}<\infty$.

Here

$$
\hat{\varphi}(t)=\int_{\mathbb{R}} \varphi(x) e^{-i t x} d x \quad(t \in \mathbb{R}) .
$$

It is also clear that if $n<n^{\prime}$ and $m<m^{\prime}$, then $T_{n, m} \subset T_{n^{\prime}, m^{\prime}}$ and that if the sequence $\left\{\varphi_{k}\right\}$ is such that $\varphi_{k} \rightarrow \varphi$ as $k \rightarrow \infty$ in $T_{n, m}$, then $\varphi_{k} \rightarrow \varphi$ as $k \rightarrow \infty$ in $T_{n^{\prime}, m^{\prime}}$. For this reason it is possible to define the countable union space containing all the spaces $T_{n, m}$ where $n, m$ vary over the set of natural numbers. In view of Theorem 2.3 and Definition 2.4 above, it easily follows that this countable union space is identical with $S_{\alpha}^{\beta}$.

Definition 2.5. (1) A sequence of functions $\left\{\varphi_{n}\right\}$ in $S_{\alpha}^{\beta}$ is said to converge to a function $\varphi \in S_{\alpha}^{\beta}$, if there exist $n^{\prime}$ and $m^{\prime}$ in $\mathbb{N}$ such that all $\varphi_{n}$ and $\varphi$ are in $T_{n^{\prime}, m^{\prime}}$ and $\varphi_{n} \rightarrow \varphi$ in $T_{n^{\prime}, m^{\prime}}$ as $n \rightarrow \infty$. This means that $\left\|\varphi_{n}-\varphi\right\|_{n^{\prime}, m^{\prime}} \rightarrow$ 0 as $n \rightarrow \infty$.

(2) By a continuous linear functional on the countable union space $S_{\alpha}^{\beta}=$ $\bigcup_{n, m} T_{n, m}$, we mean a linear functional on $S_{\alpha}^{\beta}$ which is continuous on every space $T_{n, m}$. The set of all continuous linear functionals on the above countable union space will be denoted by $\left(S_{\alpha}^{\beta}\right)^{\prime}$.

Note 2.6. The above definitions are analogous to the definitions of a continuous linear functional and the dual $\left(S_{\alpha}^{\beta}\right)^{\prime}$ of the countable union space $S_{\alpha}^{\beta}$ described in [3].

Using the equivalence of sup-norm and $L^{2}$-norm on the space $S_{\alpha, A}^{\beta, B}$ and Landau type inequalities, one can prove (this involves detailed computations which are routine and hence are omitted) the following.

(i) Given natural numbers $n, m$ and $\varphi \in T_{n, m}$, we can find $A, B$ such that $\|\varphi\|_{\delta, \rho} \leq C\|\varphi\|_{n, m}$ for every $\delta, \rho \in(0,1]$ where $\|\varphi\|_{\delta, \rho}$ refers to the semi-norms defined on $S_{\alpha, A}^{\beta, B}$ as given in [3].

(ii) Given $A, B(A, B=1,2,3, \ldots)$ and $\varphi \in S_{\alpha, A}^{\beta, B}$, we can find $n$ and $m$ such that $\|\varphi\|_{n, m} \leq C^{\prime}\|\varphi\|_{\delta, \rho}$ for every $\delta, \rho \in(0,1]$.

In view of the above observations the following remarks are obvious.

(i) The dual of $S_{\alpha}^{\beta}$ as described in [3] is identical with the dual of $S_{\alpha}^{\beta}$ as given in our Definition 2.5. 
(ii) The countable union of the spaces $T_{n, m}$ (which is $S_{\alpha}^{\beta}$ ) is also complete. However, there is a vital difference between the countable union space described here and the one given in [3]. On the one hand, the spaces $S_{\alpha, A}^{\beta, B}$ given in [3] are Fréchet spaces whereas on the other, we have chosen the spaces $T_{n, m}$ as merely normed spaces.

Lemma 2.7. Every function in $G$ can be regarded as a continuous linear functional on $S_{\alpha}^{\beta}$, i.e. each element of $G$ can be considered as a regular element of the dual space $\left(S_{\alpha}^{\beta}\right)^{\prime}$.

Proof. Let $f \in G$. We show that $f$ can be considered as a continuous linear functional on $T_{n, m}$ for each $n, m \in \mathbb{N}$ as follows:

$$
f(\varphi)=\int_{\mathbb{R}} f(x) \varphi(x) d x \quad\left(\varphi \in T_{n, m}\right) .
$$

Using canonical estimates, it is an easy exercise to show that $f$ defines a continuous linear functional on $T_{n, m}$. We leave the details.

For the construction of our Boehmian space, we need functions with compact support, inside $S_{\alpha}^{\beta}$. But this will happen when $\beta>1$. (Note that when $\beta>1, S_{0}^{\beta}$ contains functions of bounded support (see [3]) and $S_{0}^{\beta} \subset S_{\alpha}^{\beta}$, for any $\alpha>0$ ). For this reason, hereafter we shall consider spaces $S_{\alpha}^{\beta}$ only for $\beta>1$ unless explicitly specified otherwise.

Definition 2.8. A function $\varphi: \mathbb{R} \rightarrow \mathbb{C}$ is said to be in the space $S$ if $\varphi$ is in $\mathscr{D} \cap S_{\alpha}^{\beta}$.

It is easy to verify that if $\varphi \in S$, then $\varphi \in T_{n, m} \cap G$ for each $n \in \mathbb{N}$ and some $m \in \mathbb{N}$.

Definition 2.9. Let $f \in G$ and $\varphi \in S$. The convolution of $f$ and $\varphi$ is defined as

$$
(f * \varphi)(x)=\int_{\mathbb{R}} f(x-t) \varphi(t) d t \quad(x \in \mathbb{R}) .
$$

Clearly the integral in the above definition exists and hence $f * \varphi$ is well-defined. Further the $q$ th derivative of $f * \varphi$ exists and is defined by

$$
(f * \varphi)^{(q)}(x)=\left(f^{(q)} * \varphi\right)(x) .
$$

Definition 2.10. Let $u \in\left(S_{\alpha}^{\beta}\right)^{\prime}$ and $\varphi \in S$. We define the convolution product of $u$ and $\varphi$ in the canonical way by

$$
(u * \varphi)(x)=u\left(\tau_{x} \check{\varphi}\right) \quad(x \in \mathbb{R})
$$

where $\check{\varphi}(t)=\varphi(-t)$ and $\left(\tau_{x} \varphi\right)(t)=\varphi(x-t)$. 
Note that if $\varphi \in T_{n, m}$, then for any $x \in \mathbb{R}, \tau_{x} \check{\varphi}$ is in $T_{n^{\prime}, m}$ with $n^{\prime} \geq n k$ where

$$
k= \begin{cases}2^{\left(\frac{1}{\alpha}-1\right)} & \text { for } \alpha<1 \\ 1 & \text { for } \alpha \geq 1 .\end{cases}
$$

In particular, if $\varphi \in S$, then for any $x \in \mathbb{R}, \tau_{x} \check{\varphi} \in S$.

Lemma 2.11. Let $f \in G, \varphi \in S$. Then $f * \varphi \in G$ and if further $f \in S$, then $f * \varphi \in S$ and $f * \varphi=\varphi * f$.

Proof. Follows easily using simple computations. We leave the details.

Theorem 2.12. Let $u \in\left(S_{\alpha}^{\beta}\right)^{\prime}, \varphi \in S$. Then $u * \varphi \in G$.

Proof. Since $\varphi \in S, \varphi$ is in $T_{n_{1}, m_{1}}$ for some $n_{1}, m_{1} \in \mathbb{N}$. (Note that $\varphi$ is in $T_{n_{1}, m_{1}}$ for all $n_{1} \in \mathbb{N}$ ). Choose $n^{\prime} \geq n_{1} k$ and $m^{\prime} \geq m_{1}$ with $k$ as in (1) so that $\tau_{x} \check{\varphi} \in T_{n^{\prime}, m^{\prime}}$ for all $x \in \mathbb{R}$.

Indeed, using standard arguments and computations we can easily prove the following:

(i) If $x_{n} \rightarrow x$ as $n \rightarrow \infty$, then $\left\|\tau_{x_{n}} \check{\varphi}-\tau_{x} \check{\varphi}\right\|_{n^{\prime}, m^{\prime}} \rightarrow 0$ as $n \rightarrow \infty$.

(ii) $u * \varphi$ is infinitely differentiable.

(iii) $\left|(u * \varphi)^{(q)}(x)\right| \leq c_{n, q} e^{\frac{1}{n}|x|^{1 / \alpha}} \quad(n \in \mathbb{N}, q=0,1,2, \ldots$ and $x \in \mathbb{R})$.

Using the above steps, we see that $u * \varphi \in G$, completing the proof.

3. Construction of Boehmians. The general construction of Boehmians is given in detail in the literature (see [6], [7], [8]). We shall briefly recall this construction for the sake of completeness. Let $G$ be an additive commutative semi group, $S \subset G$ and $*: G \times S \rightarrow G$ satisfy

(i) If $\varphi, \psi \in S$, then $\varphi * \psi \in S$ and $\varphi * \psi=\psi * \varphi$.

(ii) If $f \in G$ and $\varphi, \psi \in S$, then $(f * \varphi) * \psi=f *(\varphi * \psi)$.

(iii) If $f, g \in G$ and $\varphi \in S$, then $(f+g) * \varphi=f * \varphi+g * \varphi$.

Let $\Delta$ be the class of all sequences $\left(\varphi_{n}\right)$ in $S$ (called delta sequences) satisfying

(i) If $f, g \in G,\left(\varphi_{n}\right) \in \Delta$ and $f * \varphi_{n}=g * \varphi_{n}$ for all $n$, then $f=g$.

(ii) If $\left(\varphi_{n}\right),\left(\psi_{n}\right) \in \Delta$, then $\left(\varphi_{n} * \psi_{n}\right) \in \Delta$.

Let $\mathscr{A}$ denote the collection of all pairs of sequences $\left(\left(f_{n}\right),\left(\varphi_{n}\right)\right)$ where $f_{n} \in G(n \in \mathbb{N})$ and $\left(\varphi_{n}\right) \in \Delta$ satisfying the following property

$$
f_{n} * \varphi_{m}=f_{m} * \varphi_{n} \text { for all } n, m \in \mathbb{N} .
$$

Each element of $\mathscr{A}$ is called a quotient. Define a relation $\sim$ (which can be easily verified as an equivalence relation) on $\mathscr{A}$ by

$$
\left(\left(f_{n}\right),\left(\varphi_{n}\right)\right) \sim\left(\left(g_{n}\right),\left(\psi_{n}\right)\right) \Longleftrightarrow f_{n} * \psi_{m}=g_{m} * \varphi_{n} \quad \forall n, m \in \mathbb{N} .
$$


Let $\mathcal{B}(G, \Delta)=\mathscr{A} / \sim$, the set of all equivalence classes in $\mathscr{A}$. Elements of $\mathcal{B}(G, \Delta)$ are called Boehmians and a typical element containing $\left(\left(f_{n}\right),\left(\varphi_{n}\right)\right)$ is denoted by $\left[\frac{f_{n}}{\varphi_{n}}\right]$.

Now we construct the Boehmian space $\mathcal{B}(G, \Delta)$, where $G$ and $S$ are as in Section 2. The convolution product defines a map

$$
\text { * : } G \times S \rightarrow G
$$

having the required properties (easy to verify).

Let $\Delta$ be the class of all sequences $\left(\delta_{n}\right)$ in $S$ satisfying the conditions:

$$
\begin{gathered}
\int_{\mathbb{R}} \delta_{n}(x) d x=1 \quad(n \in \mathbb{N}) \\
\int_{\mathbb{R}}\left|\delta_{n}(x)\right| d x \leq M \quad(n \in \mathbb{N})
\end{gathered}
$$

(4) $\lim _{n \rightarrow \infty} \int_{|x| \geq \epsilon}\left(e^{\left(\frac{1}{m}|x|^{1 / \alpha}\right)}-1\right)\left|\delta_{n}(x)\right| d x=0$, for each $m \in \mathbb{N}$ and $\epsilon>0$.

We now show that the class $\Delta$ satisfies the following required properties of "Delta sequences".

Lemma 3.1. Let $\left(\varphi_{n}\right) \in \Delta$. For each fixed $m \in \mathbb{N}$ and $\epsilon>0$, the condition

$$
\lim _{n \rightarrow \infty} \int_{|x| \geq \epsilon}\left(e^{\left(\frac{1}{m}|x|^{1 / \alpha}\right)}-1\right)\left|\varphi_{n}(x)\right| d x=0
$$

is equivalent to

$$
\lim _{n \rightarrow \infty} \int_{\mathbb{R}}\left(e^{\left(\frac{1}{m}|x|^{1 / \alpha}\right)}-1\right)\left|\varphi_{n}(x)\right| d x=0 .
$$

Proof. Let $\eta>0$ and fix $m \in \mathbb{N}$. It suffices to prove that

$$
\begin{aligned}
\lim _{n \rightarrow \infty} \int_{|x| \geq \epsilon}\left(e^{\left(\frac{1}{m}|x|^{1 / \alpha}\right)}-1\right)\left|\varphi_{n}(x)\right| d x=0 \\
\\
\Longrightarrow \lim _{n \rightarrow \infty} \int_{\mathbb{R}}\left(e^{\left(\frac{1}{m}|x|^{1 / \alpha}\right)}-1\right)\left|\varphi_{n}(x)\right| d x=0 .
\end{aligned}
$$

Assume

$$
\lim _{n \rightarrow \infty} \int_{|x| \geq \epsilon}\left(e^{\left(\frac{1}{m}|x|^{1 / \alpha}\right)}-1\right)\left|\varphi_{n}(x)\right| d x=0 \quad(\forall \epsilon>0) .
$$

Choose $\delta>0$ such that

$$
e^{\frac{1}{m}|x|^{1 / \alpha}}-1<\frac{\eta}{2 M} \text { whenever }|x|<\delta
$$


where $M$ is as in (3). Now

$$
\begin{aligned}
& \int_{\mathbb{R}}\left(e^{\left(\frac{1}{m}|x|^{1 / \alpha}\right)}-1\right)\left|\varphi_{n}(x)\right| d x \\
& \quad \int_{|x|<\delta}\left(e^{\left(\frac{1}{m}|x|^{1 / \alpha}\right)}-1\right)\left|\varphi_{n}(x)\right| d x+\int_{|x| \geq \delta}\left(e^{\left(\frac{1}{m}|x|^{1 / \alpha}\right)}-1\right)\left|\varphi_{n}(x)\right| d x \\
& \quad<\frac{\eta}{2}+\frac{\eta}{2}=\eta
\end{aligned}
$$

for sufficiently large $n$. This completes the proof.

Theorem 3.2. If $f \in G$ and $\left(\varphi_{n}\right) \in \Delta$, then $f * \varphi_{n} \rightarrow f$ as $n \rightarrow \infty$ in $G$.

Proof. We have to prove that $P_{m, p}\left(f * \varphi_{n}-f\right) \rightarrow 0$ as $n \rightarrow \infty$ for each $m \in \mathbb{N}$ and $p=0,1,2, \ldots$ Fix $m, p$ and a constant $K>0$. Choose a compact set $H$ such that for $x \notin H$,

$$
e^{\left(\frac{1}{m^{\prime}}-\frac{1}{m}\right)|x|^{1 / \alpha}}<\frac{\epsilon}{4 K},
$$

where $m^{\prime} \geq m k$ (with $k$ as in (1)). Using the uniform continuity of $f^{(q)}$ $(q=0,1, \ldots)$ on a compact set larger than $H$, say $H_{1}$, we can get a $\delta>0$ such that whenever $|y|<\delta$ and $x \in H_{1}$, we have

$$
\left|f^{(q)}(x-y)-f^{(q)}(x)\right|<\frac{\epsilon}{4 M} .
$$

Fix this $\delta>0$. For $|x| \geq \delta$, we have

$$
e^{\frac{1}{m}|x|^{1 / \alpha}}+1 \leq C\left(e^{\frac{1}{m}|x|^{1 / \alpha}}-1\right)
$$

where $C=\frac{2}{1-e^{\frac{-1}{m}|\delta|^{1 / \alpha}}}$, which can be easily verified.

We now consider

$$
\begin{aligned}
P_{m, p}\left(f * \varphi_{n}-f\right) \leq & \sup _{x \in \mathbb{R}, q \leq p} e^{\frac{-1}{m}|x|^{1 / \alpha}} \int_{\mathbb{R}}\left|f^{(q)}(x-y)-f^{(q)}(x)\right|\left|\varphi_{n}(y)\right| d y \\
= & \sup _{x \in H_{1}, q \leq p} e^{\frac{-1}{m}|x|^{1 / \alpha}} \int_{\mathbb{R}}\left|f^{(q)}(x-y)-f^{(q)}(x)\right|\left|\varphi_{n}(y)\right| d y \\
& +\sup _{x \notin H_{1}, q \leq p} e^{\frac{-1}{m}|x|^{1 / \alpha}} \int_{\mathbb{R}}\left|f^{(q)}(x-y)-f^{(q)}(x)\right|\left|\varphi_{n}(y)\right| d y \\
= & I_{1}+I_{2},
\end{aligned}
$$

say. Hence

$$
P_{m, p}\left(f * \varphi_{n}-f\right) \leq I_{1}+I_{2} .
$$


Now the integral $I_{1}$ can be split as a sum of two integrals, say $J_{1}$ and $J_{2}$, where

$$
J_{1}=\sup _{x \in H_{1}, q \leq p} e^{-\frac{1}{m}|x|^{1 / \alpha}} \int_{|y|<\delta}\left|f^{(q)}(x-y)-f^{(q)}(x)\right|\left|\varphi_{n}(y)\right| d y
$$

and

$$
J_{2}=\sup _{x \in H_{1}, q \leq p} e^{\frac{-1}{m}|x|^{1 / \alpha}} \int_{|y| \geq \delta}\left|f^{(q)}(x-y)-f^{(q)}(x)\right|\left|\varphi_{n}(y)\right| d y .
$$

Using the uniform continuity of $f^{(q)}$ on $H_{1}$, we have

$$
J_{1} \leq \frac{\epsilon}{4 M} \int_{|y|<\delta}\left|\varphi_{n}(y)\right| d y \leq \frac{\epsilon}{4} .
$$

Note that for any integer $m^{\prime}$,

$$
\begin{aligned}
\left|f^{(q)}(x-y)-f^{(q)}(x)\right| & \leq\left|f^{(q)}(x-y)\right|+\left|f^{(q)}(x)\right| \\
& \leq C_{m^{\prime}, q}\left(e^{\frac{1}{m^{\prime}}|x-y|^{1 / \alpha}}+e^{\frac{1}{m^{\prime}}|x|^{1 / \alpha}}\right) \\
& \left.\leq C_{m^{\prime}, q}\left(e^{\frac{k}{m^{\prime}}\left(|x|^{1 / \alpha}+|y|^{1 / \alpha}\right.}\right)+e^{\frac{1}{m^{\prime}}|x|^{1 / \alpha}}\right) .
\end{aligned}
$$

Take $m^{\prime} \geq m k$ (with $k$ as in (1)) in equation (7) and use this estimate and (5) in $J_{2}$ to get

$$
\begin{aligned}
J_{2} & \leq \sup _{x \in H_{1}, q \leq p} e^{\left(\frac{1}{m^{\prime}}-\frac{1}{m}\right)|x|^{1 / \alpha}} \int_{|y| \geq \delta} C_{m^{\prime}, q}\left(e^{\frac{1}{m^{\prime}}|y|^{1 / \alpha}}+1\right)\left|\varphi_{n}(y)\right| d y \\
& \leq C C_{m^{\prime}, p} \int_{|y| \geq \delta}\left(e^{\frac{1}{m_{1}}|y|^{1 / \alpha}}-1\right)\left|\varphi_{n}(y)\right| d y \\
& <\frac{\epsilon}{4}
\end{aligned}
$$

for sufficiently large $n$. Thus

$$
I_{1} \rightarrow 0 \quad \text { as } \quad n \rightarrow \infty .
$$

It is now easy to see that

$$
I_{2} \leq \sup _{x \notin H_{1}, q \leq p} e^{\left(\frac{1}{m^{\prime}}-\frac{1}{m}\right)|x|^{1 / \alpha}} \int_{\mathbb{R}} C_{m^{\prime}, q}\left(e^{\frac{1}{m^{\prime}}|y|^{1 / \alpha}}+1\right)\left|\varphi_{n}(y)\right| d y
$$

with $m^{\prime} \geq m k$. By a standard argument we can now show that

$$
I_{2} \rightarrow 0 \quad \text { as } n \rightarrow \infty .
$$

The theorem follows from (6), (8) and (9). 
Theorem 3.3. Let $f, g \in G$ and $\left(\varphi_{n}\right) \in \Delta$. If $f * \varphi_{n}=g * \varphi_{n}$ for all $n \in \mathbb{N}$, then $f=g$ in $G$.

Proof. Follows from Theorem 3.2.

Theorem 3.4. Let $\left(\varphi_{n}\right),\left(\psi_{n}\right)$ be any two sequences in $\Delta$. Then $\left(\varphi_{n} * \psi_{n}\right)$ is also a sequence in $\Delta$.

Proof. Using Fubini's theorem (wherever necessary), the properties of the class $\Delta$, Lemma 3.1 and some standard arguments, the result follows. We leave the details.

Theorem 3.3 and Theorem 3.4 together prove that the class $\Delta$ can be used as "Delta sequences" for the construction of our Boehmian space. The resulting Boehmian space will be denoted as $\mathcal{B}(G, \Delta)$.

Definition 3.5. Let $G^{\prime}=\left(S_{\beta}^{\alpha}\right)^{\prime}$ and $S^{\prime}=\hat{S}$ where $\hat{S}$ denotes the set of all Fourier transforms of elements of $S=\mathscr{D} \cap S_{\alpha}^{\beta}$. The map $G^{\prime} \times S^{\prime}$ to $G^{\prime}$ is taken as the canonical product defined as follows:

$$
(u \psi)(\phi)=u(\psi \phi) \quad\left(\phi \in S_{\beta}^{\alpha}, u \in G^{\prime} \text { and } \psi \in S^{\prime}\right) .
$$

Let

$$
\Delta^{\prime}=\hat{\Delta}=\left\{\left(\hat{\varphi}_{n}\right):\left(\varphi_{n}\right) \in \Delta\right\} .
$$

Instead of equipping $\left(S_{\alpha}^{\beta}\right)^{\prime}$ with the canonical weak* convergence, we shall define a different notion of convergence as follows.

Definition 3.6. A sequence $\left\{u_{n}\right\}$ in $\left(S_{\alpha}^{\beta}\right)^{\prime}$ converges to $u \in\left(S_{\alpha}^{\beta}\right)^{\prime}$, if for every $m, k \in \mathbb{N}$ there exists a sequence $\left\{c_{n, m, k}\right\}$ with $c_{n, m, k} \rightarrow 0$ as $n \rightarrow \infty$ such that for all $\psi \in T_{m, k} \subset S_{\alpha}^{\beta}$,

$$
\left|\left(u_{n}-u\right)(\psi)\right| \leq c_{n, m, k}|| \psi \|_{m, k} .
$$

Hereafter convergence in $\left(S_{\alpha}^{\beta}\right)^{\prime}$ means the above convergence only, unless stated otherwise.

Theorem 3.7. $u_{n} \rightarrow u$ as $n \rightarrow \infty$ in $\left(S_{\alpha}^{\beta}\right)^{\prime}$ if and only if $\hat{u}_{n} \rightarrow \hat{u}$ as $n \rightarrow \infty$ in $\left(S_{\beta}^{\alpha}\right)^{\prime}$.

Proof. The result follows from the definition of convergence and the facts that $\hat{u}(\varphi)=u(\hat{\varphi})\left(u \in\left(S_{\alpha}^{\beta}\right)^{\prime}, \varphi \in S_{\beta}^{\alpha}\right)$ and $\|\psi\|_{p, q}=\|\hat{\psi}\|_{q, p}\left(\psi \in S_{\alpha}^{\beta}\right)$.

Theorem 3.8. If $f_{n} \rightarrow 0$ as $n \rightarrow \infty$ in $G$, then $f_{n} \rightarrow 0$ as $n \rightarrow \infty$ in $\left(S_{\alpha}^{\beta}\right)^{\prime}$.

Proof. Since $f_{n} \rightarrow 0$ as $n \rightarrow \infty$ in $G, P_{m, q}\left(f_{n}\right) \rightarrow 0$ as $n \rightarrow \infty$ for all $m \in \mathbb{N}, q=0,1,2, \ldots$ For any $\varphi \in T_{n^{\prime}, m^{\prime}} \subset S_{\alpha}^{\beta}$, choose $m>n^{\prime}$ and consider

$$
\left|f_{n}(\varphi)\right| \leq \int_{\mathbb{R}}\left|f_{n}(x)\right||\varphi(x)| d x .
$$


A simple computation shows that

$$
\left|f_{n}(\varphi)\right| \leq c_{n}|| \varphi \|_{n^{\prime}, m^{\prime}},
$$

where

$$
c_{n}=P_{m, 0}\left(f_{n}\right) \int_{\mathbb{R}} e^{\left(\frac{1}{m}-\frac{1}{n^{\prime}}\right)|x|^{1 / \alpha}} d x \rightarrow 0 \quad \text { as } \quad n \rightarrow \infty .
$$

This shows that $f_{n} \rightarrow 0$ as $n \rightarrow \infty$ in $\left(S_{\alpha}^{\beta}\right)^{\prime}$. This proves the theorem.

Theorem 3.9. Let $u_{n} \rightarrow 0$ as $n \rightarrow \infty$ in $\left(S_{\alpha}^{\beta}\right)^{\prime}$ and $\varphi \in S$ then $u_{n} * \varphi \rightarrow 0$ as $n \rightarrow \infty$ in $G$.

Proof. Since $u_{n} \rightarrow 0$ as $n \rightarrow \infty$, for any $\psi \in T_{n^{\prime}, m^{\prime}}$ there exists a sequence $\left\{c_{n}\right\}$ (depending on $n^{\prime}$ and $m^{\prime}$ ) with $c_{n} \rightarrow 0$ as $n \rightarrow \infty$ such that

$$
\left|u_{n}(\psi)\right| \leq c_{n}\|\psi\|_{n^{\prime}, m^{\prime}} .
$$

We fix $m$ and $q$ and consider the semi-norm $P_{m, q} \cdot \varphi \in S\left(\varphi \in T_{r, s}\right.$ for all $r$ and some $s)$ implies that $\tau_{x}\left(\varphi^{(q)}\right)^{-} \in S(q=0,1,2, \ldots$ and for each fixed $x \in \mathbb{R}$ ) and hence $\tau_{x}\left(\varphi^{(q)}\right)^{-} \in T_{r^{\prime}, s^{\prime}}$ where $r^{\prime}>\max \{r, m\}$ and $s^{\prime} \geq s$. Consider

$$
\begin{aligned}
P_{m, q}\left(u_{n} * \varphi\right) & =\sup _{x \in \mathbb{R}, p \leq q}\left|u_{n}\left(\tau_{x}\left(\varphi^{(p)}\right)^{\swarrow}\right)\right| e^{\frac{-1}{m}|x|^{1 / \alpha}} \\
& \leq \sup _{x \in \mathbb{R}, p \leq q} c_{n}\left\|\tau_{x}\left(\varphi^{(p)}\right)^{-}\right\|_{r^{\prime}, s^{\prime}} e^{\frac{-1}{m}|x|^{1 / \alpha}} \\
& \leq c_{n} \sup _{x \in \mathbb{R}} e^{\left(\frac{k}{r^{\prime}}-\frac{1}{m}\right)|x|^{1 / \alpha}} \sup _{p \leq q}\left\|\varphi^{(p)}\right\|_{r^{\prime}, s^{\prime}} \\
& \leq C^{\prime} c_{n}\|\varphi\|_{r, s}
\end{aligned}
$$

(with $k$ as in (1)) where $\left\|\left(\varphi^{(q)}\right)^{\sim}\right\|_{r^{\prime}, s^{\prime}} \leq C_{p}\|\varphi\|_{r, s}$ and

$$
C^{\prime}=\sup _{x \in \mathbb{R}} e^{\left(\frac{k}{r^{\prime}}-\frac{1}{m}\right)|x|^{1 / \alpha}} \sup _{p \leq q} C_{p}<\infty .
$$

Hence

$$
P_{m, q}\left(u_{n} * \varphi\right) \leq C^{\prime} c_{n}\|\varphi\|_{r, s} .
$$

As $n \rightarrow \infty, c_{n} \rightarrow 0$ implies that $P_{m, q}\left(u_{n} * \varphi\right) \rightarrow 0$ as $n \rightarrow \infty$. This completes the proof of our theorem.

Theorem 3.10. Let $u \in\left(S_{\beta}^{\alpha}\right)^{\prime}$ and $\left(\hat{\varphi}_{n}\right) \in \Delta^{\prime}$. Then $u \hat{\varphi}_{n} \rightarrow u$ as $n \rightarrow \infty$ in $\left(S_{\beta}^{\alpha}\right)^{\prime}$.

Proof. Fix $m, k \in \mathbb{N}$. Let $\psi \in T_{m^{\prime}, k^{\prime}} \subset S_{\beta}^{\alpha}$. As $u \in\left(S_{\beta}^{\alpha}\right)^{\prime}$, there is a positive constant $C$ such that

$$
|u(\psi)| \leq C|| \psi \|_{m^{\prime}, k^{\prime}}
$$


for all $\psi \in T_{m^{\prime}, k^{\prime}}$. A simple computation shows that

$$
\left|\left(u \hat{\varphi}_{n}-u\right)(\psi)\right| \leq C|| \hat{\varphi}_{n} \psi-\psi \|_{p, q},
$$

where $p>k m^{\prime}$ and $q>k k^{\prime}$ with $k$ as in (1).

Consider

$\left\|\hat{\varphi}_{n} \psi-\psi\right\|_{p, q}=\sup _{x \in \mathbb{R}}\left|\left(\hat{\varphi}_{n} \psi\right)(x)-\psi(x)\right| e^{\frac{1}{p}|x|^{1 / \beta}}+\sup _{t \in \mathbb{R}}\left|\left(\check{\varphi}_{n} * \hat{\psi}\right)(t)-\hat{\psi}(t)\right| e^{\frac{1}{q}|t|^{1 / \alpha}}$.

Choosing $p$ and $q$ sufficiently large we have

$$
\left\|\hat{\varphi}_{n} \psi-\psi\right\|_{p, q} \leq c_{n}^{\prime}\|\psi\|_{m^{\prime}, k^{\prime}}+d_{n}^{\prime}\left\|(\hat{\psi})^{\prime}\right\|_{p^{\prime}, q^{\prime}},
$$

where

$$
c_{n}^{\prime}=\sup _{x \in \mathbb{R}}\left|\hat{\varphi}_{n}(x)-1\right| e^{\left(\frac{1}{p}-\frac{1}{m^{\prime}}\right)|x|^{1 / \beta}}
$$

and

$$
d_{n}^{\prime}=K \int_{\mathbb{R}}\left|\check{\varphi}_{n}(y)\right||y| e^{\frac{1}{m}|y|^{1 / \alpha}} d y
$$

with $m k<q, k$ as in (1) and $K$, a constant. Using simple computations we can prove that the sequences $\left\{c_{n}^{\prime}\right\}$ and $\left\{d_{n}^{\prime}\right\}$ tend to zero as $n \rightarrow \infty$. Using the continuity of differentiation, we can get that

$$
\left\|(\hat{\psi})^{\prime}\right\|_{p^{\prime}, q^{\prime}} \leq C^{\prime}\|\hat{\psi}\|_{k^{\prime}, m^{\prime}} \leq C^{\prime}\|\psi\|_{m^{\prime}, k^{\prime}}
$$

Thus, we have

$$
\left\|\hat{\varphi}_{n} \psi-\psi\right\|_{p, q} \leq c_{n}\|\psi\|_{m^{\prime}, k^{\prime}}
$$

where $c_{n}=C^{\prime} d_{n}^{\prime}+c_{n}^{\prime} \rightarrow 0$ as $n \rightarrow \infty$. This completes the proof of our theorem.

The following theorems can be easily verified. We leave the details.

Theorem 3.11. Let $v_{1}, v_{2} \in G^{\prime}$ and $\left(\sigma_{n}\right) \in \Delta^{\prime}$. If $v_{1} \sigma_{n}=v_{2} \sigma_{n}$ for all $n$, then $v_{1}=v_{2}$ in $G^{\prime}$.

Theorem 3.12. If $\left(a_{n}\right),\left(b_{n}\right) \in \Delta^{\prime}$, then $\left(a_{n} b_{n}\right) \in \Delta^{\prime}$.

Using $G^{\prime}$ and $\Delta^{\prime}$ as $G$ and $\Delta$ we can now construct a Boehmian space in a canonical way. This Boehmian space will be denoted by $\mathcal{B}\left(G^{\prime}, \Delta^{\prime}\right)$. We shall now obtain some properties of sequences in $G$ and $G^{\prime}$.

Theorem 3.13. Let $f_{n} \rightarrow f$ as $n \rightarrow \infty$ in $G$ and $\varphi \in S$. Then $f_{n} * \varphi \rightarrow f * \varphi$ as $n \rightarrow \infty$ in $G$.

Proof. Since $f_{n} \rightarrow f$ as $n \rightarrow \infty$ in $G$, for any $m \in \mathbb{N}$ and $q=0,1,2, \ldots$

$$
P_{m, q}\left(f_{n}-f\right) \rightarrow 0 \quad \text { as } \quad n \rightarrow \infty .
$$

Fix $m \in \mathbb{N}$ and $q \in \mathbb{N} \cup\{0\}$. As $\varphi \in S$, choose $r$ and $m^{\prime}$ so that $m^{\prime}>m k$ and $m^{\prime}>r k$ (with $k$ as in (1)) and $\varphi \in T_{r, s}$. 
Consider

$$
\begin{aligned}
& P_{m, q}\left(\left(f_{n}-f\right) * \varphi\right) \leq \sup _{x \in \mathbb{R}, p \leq q} \int_{\mathbb{R}}\left|\left(f_{n}-f\right)^{(p)}(y)\right||\varphi(x-y)| e^{-\frac{1}{m}|x|^{1 / \alpha}} d y \\
& \leq \sup _{x \in \mathbb{R}, p \leq q} \int_{\mathbb{R}}\left|\left(f_{n}-f\right)^{(p)}(y)\right||\varphi(x-y)| e^{-\frac{1}{m}|x|^{1 / \alpha}} e^{\frac{1}{m^{\mid}}|y|^{1 / \alpha}} e^{-\frac{1}{m^{\prime}}|y|^{1 / \alpha}} d y \\
& \leq \sup _{x \in \mathbb{R}} e^{\left(\frac{k}{m^{\prime}}-\frac{1}{m}\right)|x|^{1 / \alpha}} P_{m^{\prime}, q}\left(f_{n}-f\right) \int_{\mathbb{R}}|\varphi(x-y)| e^{\frac{k}{m^{\prime}}|x-y|^{1 / \alpha}} d y
\end{aligned}
$$

(substituting $t=x-y$ )

$$
\begin{aligned}
& \leq P_{m^{\prime}, q}\left(f_{n}-f\right) \int_{\mathbb{R}}|\varphi(t)| e^{\frac{k}{m^{\prime}}|t|^{1 / \alpha}} d t \\
& \leq P_{m^{\prime}, q}\left(f_{n}-f\right)\|\varphi\|_{r, s} \int_{\mathbb{R}} e^{\left(\frac{k}{m^{\prime}}-\frac{1}{r}\right)|t|^{1 / \alpha}} d t .
\end{aligned}
$$

Since

$$
\|\varphi\|_{r, s} \int_{\mathbb{R}} e^{\left(\frac{k}{m^{\prime}}-\frac{1}{r}\right)|t|^{1 / \alpha}} d t
$$

is finite and $P_{m^{\prime}, q}\left(f_{n}-f\right) \rightarrow 0$ as $n \rightarrow \infty$ we have

$$
P_{m, q}\left(f_{n} * \varphi-f * \varphi\right) \rightarrow 0 \quad \text { as } \quad n \rightarrow \infty .
$$

This completes the proof.

Theorem 3.14. Let $f_{n} \rightarrow f$ as $n \rightarrow \infty$ in $G$ and $\left(\varphi_{n}\right) \in \Delta$. Then $f_{n} * \varphi_{n} \rightarrow$ $f$ as $n \rightarrow \infty$ in $G$.

Proof. Since $f_{n} \rightarrow f$ as $n \rightarrow \infty$ in $G$, for any $m \in \mathbb{N}$ and $q=0,1,2, \ldots$

$$
P_{m, q}\left(f_{n}-f\right) \rightarrow 0 \text { as } n \rightarrow \infty \text {. }
$$

Also

$$
\left(f_{n} * \varphi_{n}\right)-f=\left(\left(f_{n}-f\right) * \varphi_{n}\right)+\left(f * \varphi_{n}-f\right) .
$$

By Theorem 3.2, $f * \varphi_{n} \rightarrow f$ as $n \rightarrow \infty$ in $G$. To prove the required result it is enough to prove that $\left(f_{n}-f\right) * \varphi_{n} \rightarrow 0$ as $n \rightarrow \infty$ in $G$.

Fix $m$ and $q$ and choose $m^{\prime}>m k$ ( $k$ is a constant as in (1)). Consider

$$
\begin{aligned}
P_{m, q}\left(\left(f_{n}-f\right) * \varphi_{n}\right) & =\sup _{x \in \mathbb{R}, p \leq q}\left|\left(\left(f_{n}-f\right)^{(p)} * \varphi_{n}\right)(x)\right| e^{-\frac{1}{m}|x|^{1 / \alpha}} \\
& \leq \sup _{x \in \mathbb{R}, p \leq q} e^{-\frac{1}{m}|x|^{1 / \alpha}} \int_{\mathbb{R}}\left|\left(f_{n}^{(p)}-f^{(p)}\right)(x-y)\right|\left|\varphi_{n}(y)\right| d y .
\end{aligned}
$$

A simple computation shows that

$$
P_{m, q}\left(\left(f_{n}-f\right) * \varphi_{n}\right) \leq M^{\prime} P_{m^{\prime}, q}\left(f_{n}-f\right),
$$


where

$$
M^{\prime}=\sup _{x \in \mathbb{R}} e^{\left(\frac{k}{m^{\prime}}-\frac{1}{m}\right)|x|^{1 / \alpha}} \int_{x \in \mathbb{R}}\left|\varphi_{n}(y)\right| e^{\frac{1}{m}|y|^{1 / \alpha}} d y<\infty
$$

(since $\left(\varphi_{n}\right) \in \Delta$ ). $P_{m^{\prime}, q}\left(f_{n}-f\right) \rightarrow 0$ as $n \rightarrow \infty$ implies that

$$
P_{m, q}\left(\left(f_{n}-f\right) * \varphi_{n}\right) \rightarrow 0 \quad \text { as } \quad n \rightarrow \infty .
$$

This completes the proof.

Theorem 3.15. Let $v_{n} \rightarrow v$ as $n \rightarrow \infty$ in $G^{\prime}$ and $\psi \in S^{\prime}$. Then $v_{n} \psi \rightarrow v \psi$ as $n \rightarrow \infty$ in $G^{\prime}$.

Proof. Follows easily from the Theorems 3.7, 3.8 and 3.9.

Theorem 3.16. Let $v_{n} \rightarrow v$ as $n \rightarrow \infty$ in $G^{\prime}$ and $\left(\hat{\varphi}_{n}\right) \in \Delta^{\prime}$. Then $v_{n} \hat{\varphi}_{n} \rightarrow v$ as $n \rightarrow \infty$ in $G^{\prime}$.

Proof. Since $v_{n} \rightarrow v$ as $n \rightarrow \infty$ in $G^{\prime}$, for any $\phi \in T_{m^{\prime}, n^{\prime}} \subset S_{\beta}^{\alpha}$, there exists constants $c_{n}$ depending on $m^{\prime}$ and $n^{\prime}$ with $c_{n} \rightarrow 0$ as $n \rightarrow \infty$ such that

$$
\left|\left(v_{n}-v\right)(\phi)\right| \leq c_{n}\|\phi\|_{m^{\prime}, n^{\prime}} .
$$

As $v \in G^{\prime}$, there exists a constant $C$ such that

$$
|v(\phi)| \leq C\|\phi\|_{m^{\prime}, n^{\prime}} \quad\left(\varphi \in T_{m^{\prime}, n^{\prime}}\right) .
$$

Now

$$
\left|\left(v_{n} \hat{\varphi}_{n}-v\right)(\phi)\right| \leq\left|\left(v_{n}-v\right)\left(\hat{\varphi}_{n} \phi\right)\right|+\left|v\left(\hat{\varphi}_{n} \phi-\phi\right)\right| .
$$

As in the proof of Theorem 3.10, we have

$$
\left\|\hat{\varphi}_{n} \phi-\phi\right\|_{q, p} \leq c_{n}^{\prime}\|\phi\|_{m^{\prime}, n^{\prime}} \quad \text { where } c_{n}^{\prime} \rightarrow 0 \text { as } n \rightarrow \infty
$$

and hence

$$
\left\|\hat{\varphi}_{n} \phi\right\|_{q, p} \leq\left(c_{n}^{\prime}+1\right)\|\phi\|_{m^{\prime}, n^{\prime}} .
$$

Thus from (10), (11) and (12), we have

$$
\left|\left(v_{n} \hat{\varphi}_{n}-v\right)(\phi)\right| \leq d_{n}\|\phi\|_{m^{\prime}, n^{\prime}},
$$

where $d_{n}=c_{n}\left(c_{n}^{\prime}+1\right)+C c_{n}^{\prime} \rightarrow 0$ as $n \rightarrow \infty$. This completes the proof.

Definition 3.17. A sequence of Boehmians $\left\{X_{n}\right\}$ in $\mathcal{B}(G, \Delta)$ is said to $\delta$-converge to a Boehmian $X \in \mathcal{B}(G, \Delta)$ denoted by $X_{n} \stackrel{\delta}{\rightarrow} X$, if there exists a delta sequence $\left(\delta_{k}\right) \in \Delta$ such that $X_{n} * \delta_{k}$ and $X * \delta_{k}$ are in $G$ for $k=1,2, \ldots$ and

$$
X_{n} * \delta_{k} \rightarrow X * \delta_{k}
$$

in $G$ as $n \rightarrow \infty$, for each $k=1,2,3, \ldots$.

Theorem 3.18. The mapping $T_{1}:\left(S_{\alpha}^{\beta}\right)^{\prime} \rightarrow \mathcal{B}(G, \Delta)$ defined by $T_{1}(u)=$ $\left[\frac{u * \varphi_{n}}{\varphi_{n}}\right]$ where $\left(\varphi_{n}\right) \in \Delta$ is any delta sequence, is a continuous imbedding of $\left(S_{\alpha}^{\beta}\right)^{\prime}$ in $\mathcal{B}(G, \Delta)$. 
Proof. If $u \in\left(S_{\alpha}^{\beta}\right)^{\prime}$ and $\left(\varphi_{n}\right) \in \Delta$, then by Theorem 2.12, $f_{n}=u * \varphi_{n} \in G$, for all $n$. Using the associativity of convolutions we can prove that

$$
f_{n} * \varphi_{m}=f_{m} * \varphi_{n}, \quad(m, n \in \mathbb{N}) .
$$

Hence $\left[\frac{f_{n}}{\varphi_{n}}\right] \in \mathcal{B}(G, \Delta)$. We first show that $T_{1}$ is one-one.

If $\left[\frac{u * \varphi_{n}}{\varphi_{n}}\right]=\left[\frac{v * \varphi_{n}}{\varphi_{n}}\right]$ in $\mathcal{B}(G, \Delta)$, then

$$
\begin{array}{lll}
\left(u * \varphi_{n}\right) * \varphi_{m}=\left(v * \varphi_{m}\right) * \varphi_{n} & \text { in } G & (m, n \in \mathbb{N}) . \\
\left(u * \varphi_{m}\right) * \varphi_{n}=\left(v * \varphi_{m}\right) * \varphi_{n} & \text { in } G & (m, n \in \mathbb{N}) .
\end{array}
$$

By Theorem 3.3, we get $u * \varphi_{m}=v * \varphi_{m}$ in $G$ for all $m \in \mathbb{N}$. Taking Fourier transform on both sides, we get that

$$
\hat{\varphi}_{m} \hat{u}=\hat{\varphi}_{m} \hat{v} \quad \text { in }\left(S_{\beta}^{\alpha}\right)^{\prime} \quad(m \in \mathbb{N}) .
$$

Applying Theorem 3.11 we get $\hat{u}=\hat{v}$ in $\left(S_{\beta}^{\alpha}\right)^{\prime}$ and hence $u=v$ in $\left(S_{\alpha}^{\beta}\right)^{\prime}$. This proves that $T_{1}$ is one-one.

To prove that $T_{1}$ is continuous, let us assume that $u_{m} \rightarrow 0$ as $m \rightarrow \infty$ in $\left(S_{\alpha}^{\beta}\right)^{\prime}$. Corresponding to each $u_{m} \in\left(S_{\alpha}^{\beta}\right)^{\prime}$, consider $X_{m}=\left[\frac{u_{m} * \varphi_{n}}{\varphi_{n}}\right] \in$ $\mathcal{B}(G, \Delta)$. We now prove that $X_{m} \stackrel{\delta}{\rightarrow} 0$ as $m \rightarrow \infty$ in $\mathcal{B}(G, \Delta)$. Take $\left(\varphi_{k}\right) \in \Delta$ and consider $X_{m} * \varphi_{k}=u_{m} * \varphi_{k} \in G$ for $k \in \mathbb{N}$. Since $u_{m} \rightarrow 0$ as $m \rightarrow \infty$ in $\left(S_{\alpha}^{\beta}\right)^{\prime}$. Applying Theorem 3.9 we get that

$$
u_{m} * \delta_{k} \rightarrow 0
$$

in $G$ as $m \rightarrow \infty(k \in \mathbb{N})$. This shows that $X_{m} \stackrel{\delta}{\rightarrow} 0$ as $m \rightarrow \infty$ in $\mathcal{B}(G, \Delta)$. This completes the proof of our theorem.

Theorem 3.19. The mapping $T_{2}: G^{\prime}\left(=\left(S_{\beta}^{\alpha}\right)^{\prime}\right) \rightarrow \mathcal{B}\left(G^{\prime}, \Delta^{\prime}\right)$ defined by $T_{2}(v)=\left[\frac{v \hat{\varphi}_{n}}{\hat{\varphi}_{n}}\right]$ where $\left(\hat{\varphi}_{n}\right) \in \Delta^{\prime}$ is any delta sequence, is a continuous imbedding of $G^{\prime}$ in $\mathcal{B}\left(G^{\prime}, \Delta^{\prime}\right)$.

Proof. Since $v \in G^{\prime}$ and $\left(\hat{\varphi}_{n}\right) \in \Delta^{\prime}, v \hat{\varphi}_{n} \in G^{\prime}$ for all $n \in \mathbb{N}$. Simple computation shows that

$$
\left(v \hat{\varphi}_{n}\right) \hat{\varphi}_{m}=\left(v \hat{\varphi}_{m}\right) \hat{\varphi}_{n} \quad \text { in } \quad G^{\prime} \quad(m, n \in \mathbb{N}) .
$$

Hence $\left[\frac{v \hat{\varphi}_{n}}{\hat{\varphi}_{n}}\right] \in \mathcal{B}\left(G^{\prime}, \Delta^{\prime}\right)$. As above in the proof of Theorem 3.18, using Theorems 3.11, 3.15 and 3.16, we can show that $T_{2}$ is a continuous one-one imbedding of $G^{\prime}$ in $\mathcal{B}\left(G^{\prime}, \Delta^{\prime}\right)$. 
4. Fourier Transform. Let $X=\left[\frac{f_{n}}{\varphi_{n}}\right] \in \mathcal{B}(G, \Delta)$. Since $f_{n} \in G, \hat{f}_{n} \in G^{\prime}$ for $n=1,2,3, \ldots$ As $\left(\varphi_{n}\right) \in \Delta,\left(\hat{\varphi}_{n}\right) \in \Delta^{\prime}$. Also $\left(\left(f_{n}\right),\left(\varphi_{n}\right)\right)$ is a quotient in $\mathcal{B}(G, \Delta)$ implies that

$$
f_{m} * \varphi_{n}=f_{n} * \varphi_{m}, \quad(m, n \in \mathbb{N}) .
$$

Taking Fourier transform on both sides, we have

$$
\hat{f}_{m} \hat{\varphi}_{n}=\hat{f}_{n} \hat{\varphi}_{m} \quad \text { in } \quad G^{\prime} \quad(m, n \in \mathbb{N}) .
$$

This proves that $\left(\left(\hat{f}_{n}\right),\left(\hat{\varphi}_{n}\right)\right)$ is a quotient in $\mathcal{B}\left(G^{\prime}, \Delta^{\prime}\right)$. We can therefore define the Fourier transform of $X($ denoted as $\hat{X})$ by $\hat{X}=\left[\frac{\hat{f}_{n}}{\hat{\varphi}_{n}}\right] \in \mathcal{B}\left(G^{\prime}, \Delta^{\prime}\right)$. It is also easy to see that this is well-defined.

Definition 4.1. Let $X=\left[\frac{f_{n}}{\varphi_{n}}\right], Y=\left[\frac{g_{n}}{\psi_{n}}\right] \in \mathcal{B}(G, \Delta)$ and $\psi \in S$. Define

(i) $X+Y=\left[\frac{f_{n} * \psi_{n}+g_{n} * \varphi_{n}}{\varphi_{n} * \psi_{n}}\right] \in \mathcal{B}(G, \Delta)$.

(ii) $\alpha X=\left[\frac{\alpha f_{n}}{\varphi_{n}}\right] \in \mathcal{B}(G, \Delta) \quad(\alpha \in \mathbb{C})$.

(iii) $X * \psi=\left[\frac{f_{n} * \psi}{\varphi_{n}}\right] \in \mathcal{B}(G, \Delta)$.

(iv) $\hat{X}+\hat{Y}=\left[\frac{\hat{\psi}_{n} \hat{f}_{n}+\hat{\varphi}_{n} \hat{g}_{n}}{\hat{\varphi}_{n} \hat{\psi}_{n}}\right] \in \mathcal{B}\left(G^{\prime}, \Delta^{\prime}\right)$.

(v) $\alpha \hat{X}=\left[\frac{\alpha \hat{f}_{n}}{\hat{\varphi}_{n}}\right] \in \mathcal{B}\left(G^{\prime}, \Delta^{\prime}\right) \quad(\alpha \in \mathbb{C})$.

(vi) $\hat{\psi} \hat{X}=\left[\frac{\hat{\psi} \hat{f}_{n}}{\hat{\varphi}_{n}}\right] \in \mathcal{B}\left(G^{\prime}, \Delta^{\prime}\right)$.

The following properties follow easily from the definitions.

Theorem 4.2. The Fourier transform $T: \mathcal{B}(G, \Delta) \rightarrow \mathcal{B}\left(G^{\prime}, \Delta^{\prime}\right)$ defined by $T(X)=\hat{X}$ is a bijective, bi-continuous mapping with the following properties.

(i) $(X+Y)^{\wedge}=\hat{X}+\hat{Y} \quad(X, Y \in \mathcal{B}(G, \Delta))$.

(ii) $(\alpha X)^{\wedge}=\alpha \hat{X} \quad(\alpha \in \mathbb{C})$.

(iii) $(X * \psi)^{\wedge}=\hat{\psi} \hat{X} \quad(\psi \in S)$.

Proof. Let us first prove that $T$ is one-one. If $\left[\frac{\hat{f}_{n}}{\hat{\varphi}_{n}}\right]=\left[\frac{\hat{g}_{n}}{\hat{\psi}_{n}}\right] \in \mathcal{B}\left(G^{\prime}, \Delta^{\prime}\right)$. Then

$$
\hat{f}_{n} \hat{\psi}_{m}=\hat{g}_{m} \hat{\varphi}_{n} \quad \text { in } \quad G^{\prime} \quad(m, n \in \mathbb{N})
$$

and taking the Fourier transform, we get

$$
f_{n} * \psi_{m}=g_{m} * \varphi_{n} \quad \text { in } \quad\left(S_{\alpha}^{\beta}\right)^{\prime} \quad(m, n \in \mathbb{N}) .
$$

Thus

Hence

$$
f_{n} * \psi_{m} * \varphi_{n}=g_{m} * \varphi_{n} * \varphi_{n} \quad \text { in } \quad G \quad(m, n \in \mathbb{N}) .
$$

$$
\left[\frac{f_{n}}{\varphi_{n}}\right]=\left[\frac{f_{n} * \varphi_{n}}{\varphi_{n} * \varphi_{n}}\right]=\left[\frac{g_{n}}{\psi_{n}}\right] \in \mathcal{B}(G, \Delta) .
$$


This proves that $T$ is one-one.

$\boldsymbol{T}$ is onto: Let $Y=\left[\frac{g_{n}}{\hat{\varphi}_{n}}\right] \in \mathcal{B}\left(G^{\prime}, \Delta^{\prime}\right)$. Since $g_{n} \in G^{\prime}$, there exists $f_{n} \in$ $\left(S_{\alpha}^{\beta}\right)^{\prime}$ such that $g_{n}=\hat{f}_{n}(n \in \mathbb{N})$. As $\left(\varphi_{n}\right) \in \Delta, f_{n} * \varphi_{n} \in G$ for $n \in \mathbb{N}$ and hence $X=\left[\frac{f_{n} * \varphi_{n}}{\varphi_{n} * \varphi_{n}}\right] \in \mathcal{B}(G, \Delta)$ such that $\hat{X}=Y$. This proves that $T$ is onto.

$\boldsymbol{T}$ is continuous: Let $X_{n} \stackrel{\delta}{\rightarrow} X$ as $n \rightarrow \infty$ in $\mathcal{B}(G, \Delta)$. Then there exists a delta sequence $\left(\delta_{k}\right) \in \Delta$ such that $X_{n} * \delta_{k}, X * \delta_{k} \in G(n, k \in \mathbb{N})$ and

$$
X_{n} * \delta_{k} \rightarrow X * \delta_{k} \quad \text { as } \quad n \rightarrow \infty \quad \text { in } \quad G \quad(k=1,2,3, \ldots) .
$$

By Theorem 3.8,

$$
X_{n} * \delta_{k} \rightarrow X * \delta_{k} \quad \text { as } \quad n \rightarrow \infty \quad \text { in } \quad\left(S_{\alpha}^{\beta}\right)^{\prime} \quad(k=1,2,3, \ldots) .
$$

By Lemma 2.7 and Theorem 3.7 and by the property ( $i i i)$ of $T$ (which can be easily verified), we have $\hat{X}_{n} \hat{\delta}_{k}=\left(X_{n} * \delta_{k}\right)^{\hat{,}}, \hat{X} \hat{\delta}_{k}=\left(X * \delta_{k}\right)^{\wedge} \in G^{\prime}(n, k \in \mathbb{N})$ and

$$
\left(X_{n} * \delta_{k}\right)^{\wedge} \rightarrow\left(X * \delta_{k}\right)^{\wedge} \quad \text { as } \quad n \rightarrow \infty \quad \text { in } \quad G^{\prime} \quad(k \in \mathbb{N})
$$

or that

$$
\hat{X}_{n} \hat{\delta}_{k} \rightarrow \hat{X} \hat{\delta}_{k} \quad \text { as } \quad n \rightarrow \infty \quad \text { in } \quad G^{\prime} \quad(k \in \mathbb{N}) .
$$

Thus $\hat{X}_{n} \stackrel{\delta}{\rightarrow} \hat{X}$ as $n \rightarrow \infty$ in $\mathcal{B}\left(G^{\prime}, \Delta^{\prime}\right)$. Hence $T$ is continuous.

$\boldsymbol{T}^{-1}$ is continuous: Let $\hat{X}_{n} \stackrel{\delta}{\rightarrow} \hat{X}$ as $n \rightarrow \infty$ in $\mathcal{B}\left(G^{\prime}, \Delta^{\prime}\right)$ where $X_{n}, X \in$ $\mathcal{B}(G, \Delta)$. Then there exists a delta sequence $\left(\hat{\epsilon}_{k}\right) \in \Delta^{\prime}$ such that $\hat{X}_{n} \hat{\epsilon}_{k}, \hat{X} \hat{\epsilon}_{k} \in$ $G^{\prime}(n, k \in \mathbb{N})$ such that

$$
\hat{X}_{n} \hat{\epsilon}_{k} \rightarrow \hat{X} \hat{\epsilon}_{k} \quad \text { as } n \rightarrow \infty \quad \text { in } \quad G^{\prime} \quad(k \in \mathbb{N}) .
$$

By Theorem 3.7 and by the property (iii) of $T$, we have

$$
\left(X_{n} * \epsilon_{k}\right),\left(X * \epsilon_{k}\right) \in\left(S_{\alpha}^{\beta}\right)^{\prime}
$$

and

$$
X_{n} * \epsilon_{k} \rightarrow X * \epsilon_{k} \quad \text { as } \quad n \rightarrow \infty \quad \text { in } \quad\left(S_{\alpha}^{\beta}\right)^{\prime} \quad(k \in \mathbb{N}) .
$$

By Theorem 3.9, we have

$$
X_{n} * \epsilon_{k} * \epsilon_{k} \rightarrow X * \epsilon_{k} * \epsilon_{k} \quad \text { as } \quad n \rightarrow \infty \quad \text { in } \quad G \quad(k \in \mathbb{N}) .
$$

Thus there exists a delta sequence $\left(\epsilon_{k} * \epsilon_{k}\right) \in \Delta$ such that

$$
X_{n} * \epsilon_{k} * \epsilon_{k}, X * \epsilon_{k} * \epsilon_{k} \in G \quad(n, k \in \mathbb{N})
$$

and

$$
X_{n} * \epsilon_{k} * \epsilon_{k} \rightarrow X * \epsilon_{k} * \epsilon_{k} \quad \text { as } \quad n \rightarrow \infty \quad \text { in } \quad G \quad(k \in \mathbb{N}) .
$$

Hence $X_{n} \stackrel{\delta}{\rightarrow} X$ as $n \rightarrow \infty$ in $\mathcal{B}(G, \Delta)$.

The remaining properties follow easily from the definitions. We prefer to omit the details. This completes the proof. 
Acknowledgement. Research of the second author is supported by U.G.C. Research Fellowship in Sciences for Meritorious Students.

\section{REFERENCES}

[1] Chung, J., Chung, S. Y. and Kim, D., A characterization of the Gelfand-Shilov spaces via Fourier transform, Prod. Amer. Math. Soc. 124 (1996), 2101-2108.

[2] Chung, S. Y., Kim, D. and Lee, S., Characterization for Beurling-Bjorck space and Schwartz space, Prod. Amer. Math. Soc. 125 (11) (1997), 3229-3234.

[3] Gelfand, I. M., Shilov, G. E., Generalized Functions, Vol. I and II, Academic Press, New York, 1967.

[4] Ishihara, T., On the structure of S space, Osaka Math. J. 13 (1961), 251-264.

[5] Kashpirovskii, A. I., Equality of the spaces $S_{\alpha}^{\beta}$ and $S_{\alpha} \cap S^{\beta}$, (English. Russian original) Funct. Anal. Appl. 14, 129 (1980); translation from Funkts. Anal. Prilozh. 14, No.2, 60 (1980).

[6] Karunakaran, V., Kalpakam, N. V., Boehmians and Fourier transform, Integral Transform. Spec. Funct. 9 (3) (2000), 197-216.

[7] Mikusinski, P., Convergence of Boehmians, Japan J. Math. 9 (1983), 159-179.

[8] Mikusinski, P., Boehmians and generalized functions, Acta. Math. Hung. 51 (1988), $271-281$.

[9] Zemanian, A. H., Distribution Theory and Transform Analysis, McGraw-Hill Book Co., New York, 1965.

V. Karunakaran

School of Mathematics

Madurai Kamaraj Universityy

Madurai, India - 625021

e-mail: vkarun_mku@yahoo.co.in

Received October 3, 2009
R. Bhuvaneswari

School of Mathematics

Madurai Kamaraj University

Madurai, India - 625021

e-mail: buvibarathi@yahoo.co.in 